\title{
Injectable Hyaluronic Acid/Poly( $\gamma$-glutamic acid) Hydrogel with Step-by-step Tunable Properties for Soft Tissue Engineering
}

\author{
Xue-Bin Ma ${ }^{\mathrm{a}, \mathrm{b}}$, Rong Yang ${ }^{\mathrm{b}}$, Kanaparedu P. C. Sekhar ${ }^{\mathrm{a}}$, and Bo Chi ${ }^{\mathrm{b}^{*}}$ \\ a Key Laboratory of Colloid and Interface Chemistry of the Ministry of Education, School of Chemistry and Chemical Engineering, Shandong University, Jinan \\ 250100, China \\ b State Key Laboratory of Materials-Oriented Chemical Engineering, College of Food Science and Light Industry, Nanjing Tech University, Nanjing 211816, China
}

Electronic Supplementary Information

\begin{abstract}
Injectable hydrogels as an important class of biomaterials have gained much attention in tissue engineering. However, their crosslinking degree is difficult to be controlled after being injected into body. As we all know, the crosslinking degree strongly influences the physicochemical properties of hydrogels. Therefore, developing an injectable hydrogel with tunable crosslinking degree in vivo is important for tissue engineering. Herein, we present a dual crosslinking strategy to prepare injectable hydrogels with step-by-step tunable crosslinking degree using Schiff base reaction and photopolymerization. The developed hyaluronic acid/poly( $\gamma$-glutamic acid) (HA/ $\gamma$-PGA) hydrogels exhibit step-bystep tunable swelling behavior, enzymatic degradation behavior and mechanical properties. Mechanical performance tests show that the storage moduli of $\mathrm{HA} / \gamma-\mathrm{PGA}$ hydrogels are all less than $2000 \mathrm{~Pa}$ and the compressive moduli are in kilopascal, which have a good match with soft tissue. In addition, NIH 3 T3 cells encapsulated in HA/ $\gamma$-PGA hydrogel exhibit a high cell viability, indicating a good cytocompatibility of HA/ $\psi$-PGA hydrogel. Therefore, the developed $\mathrm{HA} / \mathrm{\gamma}$-PGA hydrogel as an injectable biomaterial has a good potential in soft tissue engineering.
\end{abstract}

Keywords Hyaluronic acid; Poly( $\gamma$-glutamic acid); Injectable hydrogel; Tissue engineering

Citation: Ma, X. B.; Yang, R.; Sekhar, K. P. C.; Chi, B. Injectable hyaluronic acid/poly( $\gamma$-glutamic acid) hydrogel with step-by-step tunable properties for soft tissue engineering. Chinese J. Polym. Sci. 2021, 39, 957-965.

\section{INTRODUCTION}

Injectable hydrogels have attracted much attention in tissue engineering due to their unique advantages, such as filling irregular shaped defects completely, in situ corporation of bioactive molecule and the potential in minimally invasive surgical procedures. ${ }^{[1,2]}$ Generally, the crosslinking degree of injectable hydrogels is adjustable before injection, once the hydrogels or precursor solutions are injected into the body, the crosslinking degree will be difficult to be adjusted. For example, for the thermosensitive injectable hydrogels, their crosslinking degree is hardly to change due to the constant body temperature. ${ }^{[3]}$ While for the covalent crosslinked injectable hydrogels (e.g., Schiff base reaction, click chemistry, Michael addition reaction), their crosslinking degree can only be adjusted before gelation by changing polymer concentration, content of reactive active groups, molar ratio of reactive groups, etc. ${ }^{[4-6]}$ It is well known that the crosslinking degree has a strong effect on the physicochemical properties of hydrogel (e.g., swelling behavior, degradation behavior, mechanical properties). Therefore, it is important to develop an injectable hydrogel with tunable

* Corresponding author, E-mail: chibo@njtech.edu.cn

Received December 2, 2020; Accepted January 25, 2021; Published online April 30, 2021 crosslinking degree in vivo for tissue engineering.

Among the covalent chemistry, photopolymerization has gained more attention in the preparation of injectable biomaterials because of its spatial and temporal adjustability. ${ }^{[7,8]}$ Their crosslinking degree can be conveniently adjusted by the ultraviolet (UV) light. ${ }^{[8,9]}$ However, since the crosslinking network cannot be formed spontaneously, the precursor solutions are easy to diffuse at the injection site before UV irradiation. Schiff base reaction has also been widely used in biomedical applications due to the mild reaction conditions and avoidance of crosslinking agents. ${ }^{[10-12]}$ Previous reports showed that by adjusting the proportion and content of reactive groups, hydrogels could be formed within a few seconds using Schiff base reaction, showing a rapid gelation ability. ${ }^{[13]}$ Therefore, combining the advantages of photopolymerization and Schiff base reaction, we expect to develop an injectable hydrogel with step-by-step tunable crosslinking degree.

Natural polymers (e.g., polysaccharides, polypeptides) have more advantages than synthetic polymers due to the inherent biological activity, biocompatibility and biodegradability. ${ }^{[14-16]}$ Hyaluronic acid (HA) is an important component of human extracellular matrix. ${ }^{[17]}$ As a naturally existing linear polysaccharide, HA possesses good biodegradability, biocompatibility and biofunctionality, which makes HA and its derivatives been widely applied for drug carrier, ${ }^{[17,18]}$ tissue engin- 
eering, ${ }^{[19,20]}$ bioprinting, ${ }^{[21,22]}$ etc. Poly $(\gamma$-glutamic acid) $(\gamma$ $P G A)$ is a anionic polypeptide with a biomimetic secondary structure similar to natural proteins. ${ }^{[23,24]}$ It also has good biocompatibility and biodegradability. Moreover, previous researches have confirmed that $\gamma$-PGA-based biomaterials can improve cell adhesion and stimulate cell migration. ${ }^{[23,25]}$ Our recent work also found that $\gamma$-PGA-containing hydrogel exhibited superior tissue repair capability. ${ }^{[26,27]}$ Hence, $\gamma$-PGA is an excellent biomaterial for tissue engineering.

Herein, an injectable hydrogel with step-by-step tunable crosslinking degree was prepared via a dual crosslinking strategy using HA and $\gamma$-PGA. Firstly, the methacrylated HA and $\gamma$-PGA were synthesized (denoted as M-HA and $M-\gamma-P G A$ ), then the aldehyde group and hydrazide group were further introduced into $M-H A$ and $M-\gamma-P G A$, respectively (denoted as $\mathrm{OM}-\mathrm{HA}$ and $\mathrm{HM}-\gamma-\mathrm{PGA})$. As shown in Scheme 1, the primary $\mathrm{HA} / \gamma$-PGA hydrogel was rapidly formed via Schiff base reaction. Then, it was irradiated by UV light to form dual crosslinked $\mathrm{HA} / \mathrm{\gamma}$-PGA hydrogel. The physicochemical properties of both single and dual crosslinked $\mathrm{HA} / \gamma$-PGA hydrogels (such as swelling, degradation, mechanical properties, etc.) were studied. Finally, the cytocompatibility of NIH 3 T3 cells in $\mathrm{HA} / \gamma$-PGA hydrogel was explored.

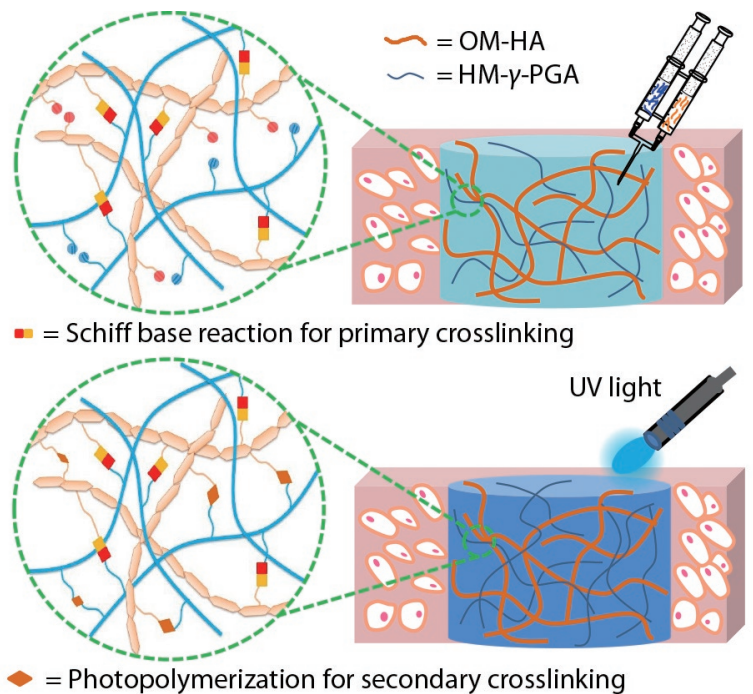

Scheme 1 Schematic illustration of single and dual crosslinked $\mathrm{HA} / \gamma$-PGA hydrogels. OM-HA and HM- $\gamma$-PGA solutions were added into two syringes, respectively, then they were injected by a dual chamber syringe equipped with a mixing tip for tissue engineering.

\section{EXPERIMENTAL}

\section{Modification of Polymers}

To prepare $M-H A$ and $M-\gamma-P G A$, the glycidyl methacrylate (GMA) reacted with $\mathrm{HA}$ and $y$-PGA under acidic conditions according to previous report. ${ }^{[28]}$ The percentages of methacrylate functionalization were $29 \%$ and $17.5 \%$, respectively. Through the oxidation of M-HA using sodium periodate, we prepared OM-HA with $16.5 \%$ oxidation degree. ${ }^{[29]} \mathrm{HM}-\gamma-\mathrm{PGA}$ was synthesized via a carbodiimide coupling reaction between adipic acid dihydrazide (ADH) and $\mathrm{M}-\gamma-\mathrm{PGA}$, and the hydrazide functionalization was $32 \%{ }^{[4]}$ The detailed experimental procedures are described in the electronic supplementary information (ESI).

\section{Preparation of Hydrogels}

For the preparation of dual crosslinked hydrogels, the individual solutions of OM-HA and $\mathrm{HM}-\gamma-\mathrm{PGA}$ were prepared in PBS $(10 \mathrm{mmol} / \mathrm{L}, \mathrm{pH}=7.4)$ containing $0.2 \mathrm{wt} \%$ of 2 -hydroxy-4'-(2hydroxyethoxy)-2-methylpropiophenone $\left(\mathrm{I}_{2959}\right)$, respectively. Then, the individual solutions were mixed under continuous agitation to form single crosslinked hydrogels (molar ratio of $-\mathrm{NHNH}_{2} /-\mathrm{CHO}$ was $1 / 1$ ). Afterwards, the formed single crosslinked hydrogels were further irradiated for $3 \mathrm{~min}$ under UV light at $365 \mathrm{~nm}\left(5 \mathrm{~mW} / \mathrm{cm}^{2}\right.$, OMRON, Japan) to prepare dual crosslinked hydrogels.

\section{Characterization of Hydrogels}

Scanning electron microscopy (SEM, Hitachi, SU1510, Japan) was used to study the morphologies of hydrogels. The hydrogel samples were frozen at $-80{ }^{\circ} \mathrm{C}$ and vacuum lyophilized at $-50{ }^{\circ} \mathrm{C}$ for $48 \mathrm{~h}$. Prior to imaging the samples were coated with gold.

Gelation time of single crosslinked hydrogels was tested by a tube inversion method. In a vial, the OM-HA and HM- $\gamma$-PGA solutions were mixed, and the gelation time was denoted as the period from the beginning to the point when the mixture stopped flowing.

The swelling behavior was tested in PBS $(10 \mathrm{mmol} / \mathrm{L}$, $\mathrm{pH}=7.4)$ at $37^{\circ} \mathrm{C}$. Firstly, a small amount of hydrogel sample was freeze-dried and weighed $\left(W_{\mathrm{d}}\right)$. Then it was dipped in PBS, and at different time intervals, the weight of swollen hydrogel $\left(W_{s}\right)$ was noted after removing the surface water. The swelling ratio of hydrogel was denoted as $\left(W_{\mathrm{s}}-W_{\mathrm{d}}\right) / W_{\mathrm{d}}$. The crosslinking degree of $\mathrm{HA} / \gamma$-PGA hydrogel was evaluated by the swelling ratio of hydrogel at equilibrium.

For the enzymatic degradation, a small amount of hydrogel was freeze-dried and the weight $\left(W_{0}\right)$ of dried hydrogel was noted. Then it was incubated in a PBS solution containing hyaluronidase $(0.1 \mathrm{mg} / \mathrm{mL})$ and papain $(0.25 \mathrm{mg} / \mathrm{mL})$ at $37^{\circ} \mathrm{C}$. At certain time intervals, the hydrogel was collected, freeze-dried, and the weight $\left(W_{t}\right)$ of freeze-dried hydrogel was noted. The weight remaining (\%) was denoted as $\left(W_{\mathrm{t}} / W_{0}\right) \times$ $100 \%$.

The OM-HA, HM- $\gamma$-PGA and freeze-dried hydrogel were used for thermal analysis. The measurement was carried out over a temperature range of $35-700^{\circ} \mathrm{C}$ by heating samples at $10^{\circ} \mathrm{C} / \mathrm{min}$ under nitrogen atmosphere on a thermogravimetry analyzer (TGA, Hengjiu, China).

The rheological and unconfined compression tests were carried out to assess the mechanical properties of hydrogels. The rheological properties were determined by using Anton Paar rheometer (MCR302, Austria) with a parallel-plate geometry ( $25 \mathrm{~mm}$ diameter) at $25^{\circ} \mathrm{C}$. For oscillatory time sweep experiments, the constant frequency and strain were fixed at $1 \mathrm{~Hz}$ and $1 \%$, respectively. For oscillatory frequency sweeps, the strain was fixed at $10 \%$. The compressive properties were evaluated by an MTS universal material testing machine (CMT2103, USA). $500 \mu \mathrm{L}$ of hydrogel samples were prepared in a cylindrical mold (diameter $=10 \mathrm{~mm}$ ), and then the compression tests were performed with a controlled rate of $10 \mathrm{~mm} / \mathrm{min}$ at $25^{\circ} \mathrm{C}$. 


\section{Cytotoxicity Assay}

Cytocompatibility of functionalized polymers was evaluated using NIH 3T3 mouse fibroblast (National platform of experimental cell resources for science-technology, Shanghai, China). Firstly, the functionalized polymers were dissolved in DMEM enriched with fetal bovine serum (FBS, V/V, 10\%) and penicillin-streptomycin $(V / V, 1 \%)$, respectively. Then the cells were seeded in a 96-well plate and incubated in an incubator (HERA cell 150i, Thermo, USA) for $12 \mathrm{~h}$. Afterwards, the medium was replaced with the prepared functionalized polymer solutions. Further cultured for $24 \mathrm{~h}$, the cell viabilities were evaluated by 3-(4,5-dimethylthiazol-2-yl)-2,5-diphenyltetrazolium bromide (MTT) assay. Later, cells were stained with acridine orange $(\mathrm{AO}) /$ ethidium bromide $(\mathrm{EB})$, and the live/dead behavior and morphology of cells after 1-, 2- and 3-days cultivation were observed by the fluorescence images.

\section{D Cell Culture}

The dual crosslinked HA/ $\gamma$-PGA hydrogel with solid content of $5 \mathrm{wt} \%$ was used to encapsulate NIH 3T3 cells for 3D cell culture. Firstly, RGD and $\mathrm{I}_{2959}$ were added into PBS $(6.7 \mathrm{mmol} / \mathrm{L}$, $\mathrm{pH}=7.0-7.2)$ at a concentration of $5 \times 10^{-3} \mathrm{~mol} / \mathrm{L}$ and $0.2 \mathrm{wt} \%$, respectively. Then, a separate $5 \mathrm{wt} \%$ solution of OM-HA and $\mathrm{HM}-\gamma-\mathrm{PGA}$ was prepared by dissolving the corresponding polymers in the above solution and passed through $0.22 \mu \mathrm{m}$ syringe filter for sterilization. Afterwards, the NIH 3 T3 cells were added into $\mathrm{HM}-\gamma$-PGA solution $\left(1 \times 10^{7}\right.$ cells $\left./ \mathrm{mL}\right)$ and then mixed with $\mathrm{OM}-\mathrm{HA}$ solution $\left(-\mathrm{NHNH}_{2} /-\mathrm{CHO}\right.$ molar ratio=1/1) to form a single crosslinked cell-laden hydrogel. Later, the dual crosslinked cell-laden hydrogel was obtained by UV irradiation for $3 \mathrm{~min}$ and carefully removed to a petri dish with DMEM medium. After incubation for $48 \mathrm{~h}$, cells were stained with AO/EB and analyzed by confocal microscopy images.

\section{Statistical Analysis}

Obtained data are shown as mean \pm standard deviation. Tukey's multiple comparison test is applied to analyze significant differences, and $p<0.05$ indicates statistical significance.

\section{RESULTS AND DISCUSSION}

\section{Modification and Characterization of Polymers}

To prepare the dual crosslinkable macromers to form hydrogels, methacrylate group was introduced onto the HA and $\gamma$-PGA backbones by the ring-opening reaction between epoxy group and the carboxyl. Then the synthesized M-HA was oxidized to introduce aldehyde group (Fig. 1a), and hydrazide group was introduced onto the M- $\gamma$-PGA backbone through a a

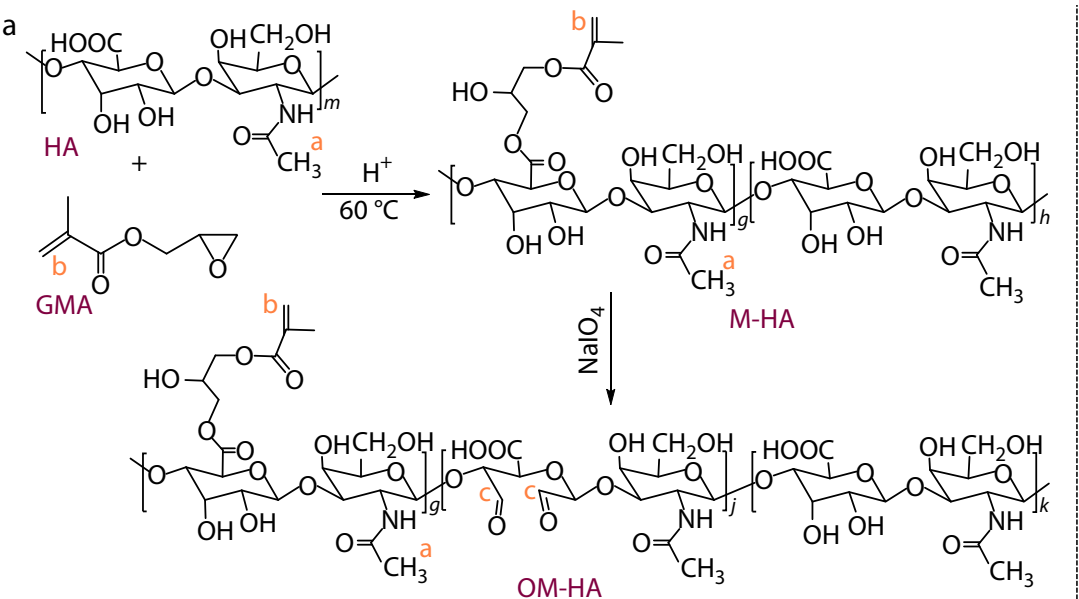

$\mathrm{OM}-\mathrm{HA}$

b<smiles>C=C(C)C(=O)OCC1CC1</smiles><smiles>CCNC(CCC=O)CC(C)C</smiles>

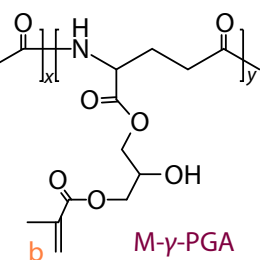

GMA

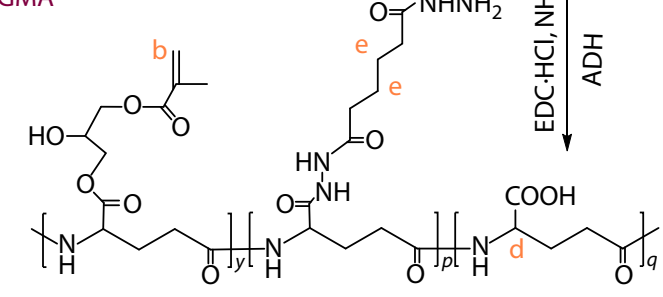

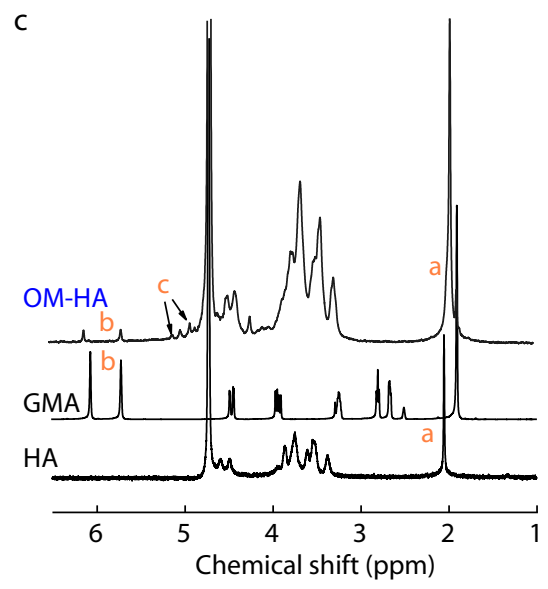

$\mathrm{d}$

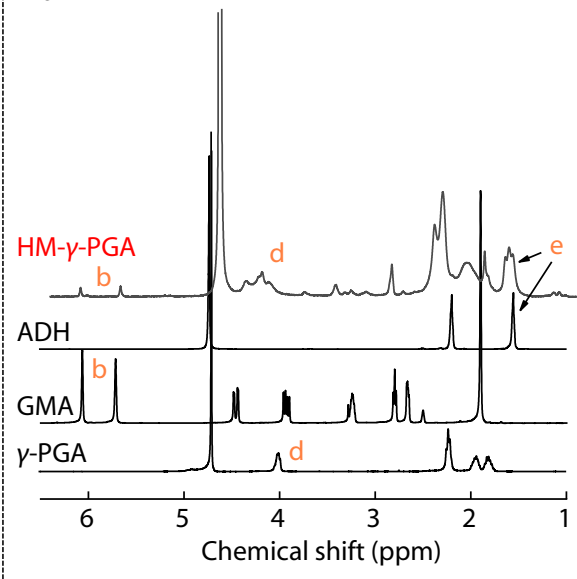

Fig. 1 Synthesis of (a) OM-HA and (b) HM- - -PGA. (c, d) ${ }^{1} \mathrm{H}-\mathrm{NMR}$ spectra of OM-HA and HM- $-\mathrm{-PGA}$. 
carbodiimide coupling reaction (Fig. 1b). Later, the structural characterizations of the synthesized polymers were confirmed by their ${ }^{1} \mathrm{H}$-NMR spectra (Figs. 1c and 1d). The characteristic peaks of vinyl group protons at $\delta 5.74-6.27$ ppm were observed in OM-HA and HM- $-\mathrm{PGA}$ spectra, which indicated that the methacrylate group was successfully grafted onto the HA and $\gamma$ PGA backbones, respectively. ${ }^{[30]}$ The aldehyde group in OM-HA was confirmed by the new peaks at $4.95-5.25 \mathrm{ppm}$ in OM-HA spectrum. ${ }^{[31,32]}$ The methylene protons at $1.46 \mathrm{ppm}$ in $\mathrm{HM}-\gamma^{-}$ PGA spectrum confirmed the successful conjugation of $A D H$ to $Y$-PGA. ${ }^{[4]}$

\section{Preparation and Characterization of Hydrogels}

The dual crosslinked hydrogels were fabricated by using Schiff base reaction and photo-initiated free radical polymerization. Firstly, OM-HA solution was mixed with HM- $y-P G A$ solution to form single crosslinked hydrogels via Schiff base reaction between aldehyde and hydrazide. The formation of single crosslinked hydrogel was studied through an oscillatory time sweep test. As shown in Fig. 2(a), in the first 100 s, the storage modulus $\left(G^{\prime}\right)$ was lower than the loss modulus $\left(G^{\prime \prime}\right)$, and the value of $G^{\prime \prime}$ was close to zero, showing a viscous fluid behavior. ${ }^{[33]}$ This fluid behavior demonstrated the injectability of mixture before gelation. The inset of Fig. 2(a) shows that the precursor solutions could be injected and mixed to form a hydrogel through a double-chamber syringe equipped with a mixing tip, indicating that the $\mathrm{HA} / \mathrm{\gamma}-\mathrm{PGA}$ hydrogel was an injectable material. The $G^{\prime}$ and $G^{\prime \prime}$ increased with an increase of time, and at $300 \mathrm{~s}$, a crossover point $\left(G^{\prime}=G^{\prime \prime}\right)$ can be observed, indicating an increase in crosslinking degree and transformation from a liquid state $\left(G^{\prime}<G^{\prime \prime}\right)$ to a gel state $\left(G^{\prime}>G^{\prime \prime}\right)$. Subsequently, $G^{\prime}$ is higher than $G^{\prime \prime}$, and both $G^{\prime}$ and $G^{\prime \prime}$ increased significantly, indicating the formation of elastic single crosslinked $\mathrm{HA} / \gamma$-PGA hydrogels. ${ }^{[33]}$ At this step, the initial crosslinking degree can be adjusted by polymer concentration. ${ }^{[13]}$ Then, the formed single crosslinked hydrogels were further crosslinked by UV light to form the dual crosslinked hydrogels.

Injectable hydrogels should gelate rapidly at injection site to avoid the diffusion of precursor. As shown in Fig. 2(b), at $3 \mathrm{wt} \%$ of solid content, the gelation time of single crosslinked
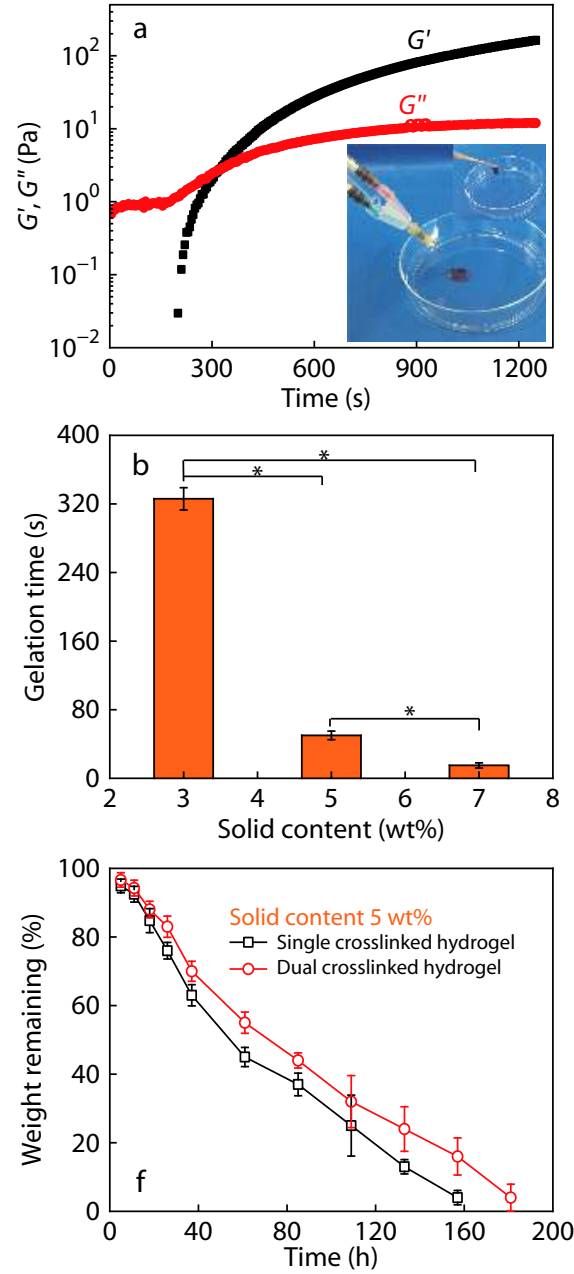
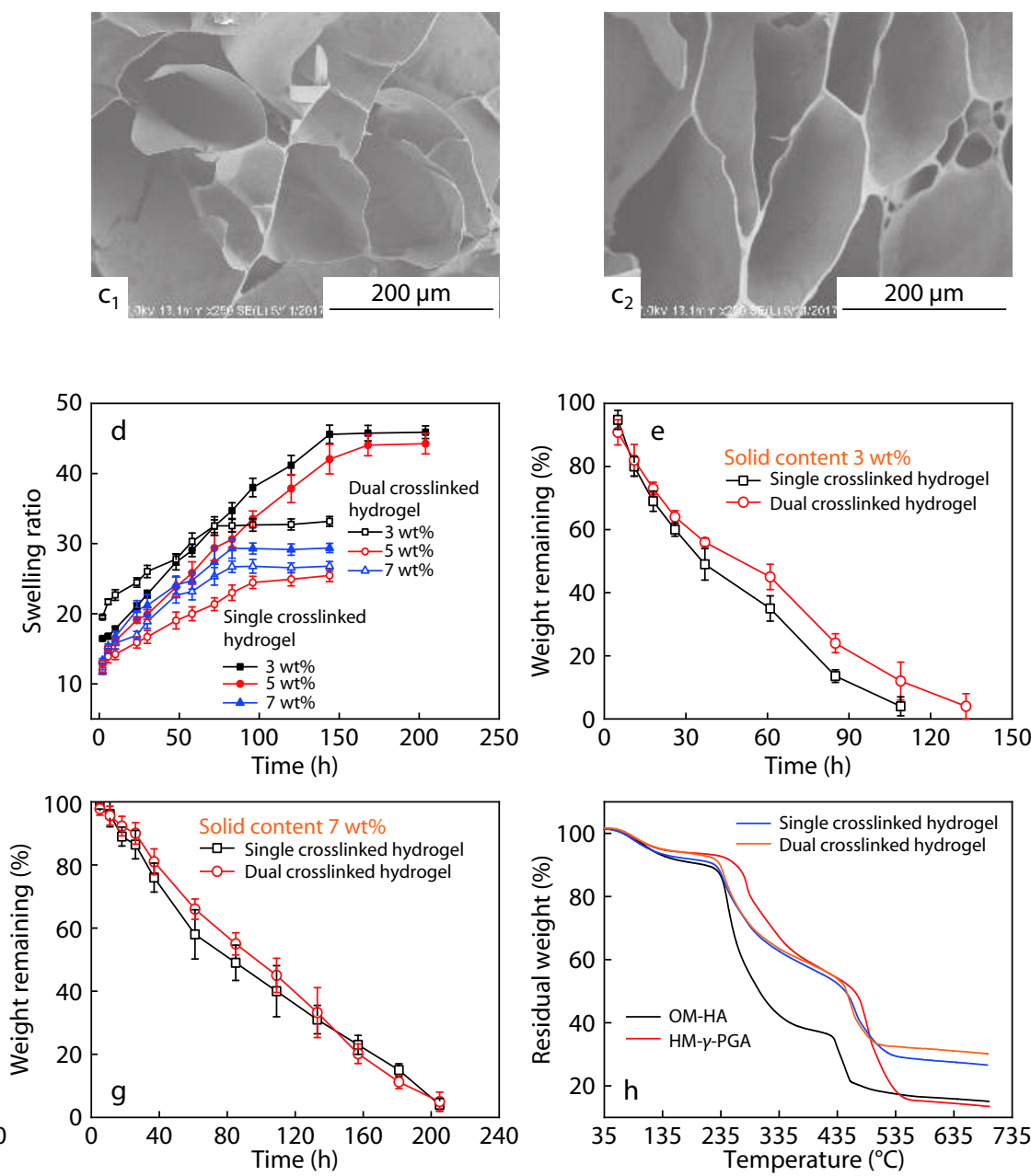

Fig. 2 (a) Time dependence of $G^{\prime}$ and $G^{\prime \prime}$ for the in situ formation of single crosslinked hydrogel with solid content of 3 wt\%, and the inset is the photos of single crosslinked hydrogel prepared by using a dual chamber syringe equipped with a mixing tip. (b) Gelation time of single crosslinked hydrogels. SEM images of freeze-dried $\left(c_{1}\right)$ single and $\left(c_{2}\right)$ dual crosslinked hydrogels with solid content of 5 wt $\%$. (d) Swelling ratio and $(\mathrm{e}-\mathrm{g})$ enzymatic degradation of single and dual crosslinked HA/ $\mathrm{H}$-PGA hydrogels. (h) TGA curves of functionalized polymers and freeze-dried $\mathrm{HA} / \gamma$-PGA hydrogels. $\left(n=3,{ }^{*} p<0.05\right)$. 
hydrogel was about $320 \mathrm{~s}$. When the solid content increased up to $5 \mathrm{wt} \%$ and $7 \mathrm{wt} \%$, the gelation time decreased to 50 and $15 \mathrm{~s}$, respectively, which indicated the controllable rapid gelation ability of single crosslinked hydrogels. The SEM images of two representative single and dual crosslinked hydrogel samples were shown in Fig. 2(c). It is clear that the single crosslinked hydrogel shows a thin layer and loose porous structure with some collapse (Fig. $2 c_{1}$ ). In contrast, the dual crosslinked hydrogel shows a denser structure, and the layer density of pores is thicker than that of single crosslinked hydrogel (Fig. $2 c_{2}$ ), which suggested a higher crosslinking degree of dual crosslinked hydrogels.

The swelling behaviors of single and dual crosslinked hydrogels are shown in Fig. 2(d). Apparently, all samples had a rapid swelling rate within the first $10 \mathrm{~h}$, later the increase in swelling rate was insignificant and gradually reached equilibrium. Compared to the single crosslinked hydrogels, the dual crosslinked hydrogel samples shows a lower swelling ratio, which indicated that the swelling behavior of $\mathrm{HA} / \gamma-\mathrm{PGA}$ hydrogel could be further adjusted by UV light. It is worth mentioning that the swelling ratios of low-concentration $\mathrm{HA} / \mathrm{Y}$ -
PGA hydrogels ( $3 w t \%$ and $5 w t \%)$ at equilibrium reduced from 45.5 to 32.5 and 44.2 to 24.5 , respectively, indicating a significant change in the crosslinking degree before and after UV irradiation. But for the high-concentration single crosslinked $\mathrm{HA} / \mathrm{\gamma}$-PGA hydrogels $(7 \mathrm{wt} \%$ ), the swelling ratios change a little (from 29.3 to 26.6) after UV irradiation, which indicated that the crosslink degree changed little. In addition, owing to the high-water sorption capacity of HA and $\gamma$-PGA, the single and dual crosslinked $\mathrm{HA} / \mathrm{\gamma}$-PGA hydrogels all exhibit high swelling levels. ${ }^{[34,35]}$ This high swelling ratio will be beneficial to cell migration, oxygen and nutrition diffusion, indicating their potential in tissue engineering. ${ }^{[36,37]}$

The degradation of single and dual crosslinked hydrogels by external enzymes was investigated in vitro at $37^{\circ} \mathrm{C}$. As shown in Figs. 2(e)-2(g), all hydrogel samples could be degraded in enzyme solutions. With the increasing solid content of hydrogels, the degradation time increased due to the increased crosslinking degree and compact network structure. ${ }^{[38]}$ Compared with the single crosslinked hydrogels, the dual crosslinked hydrogels show a longer degradation time because of the strong crosslinking state, which indicated the
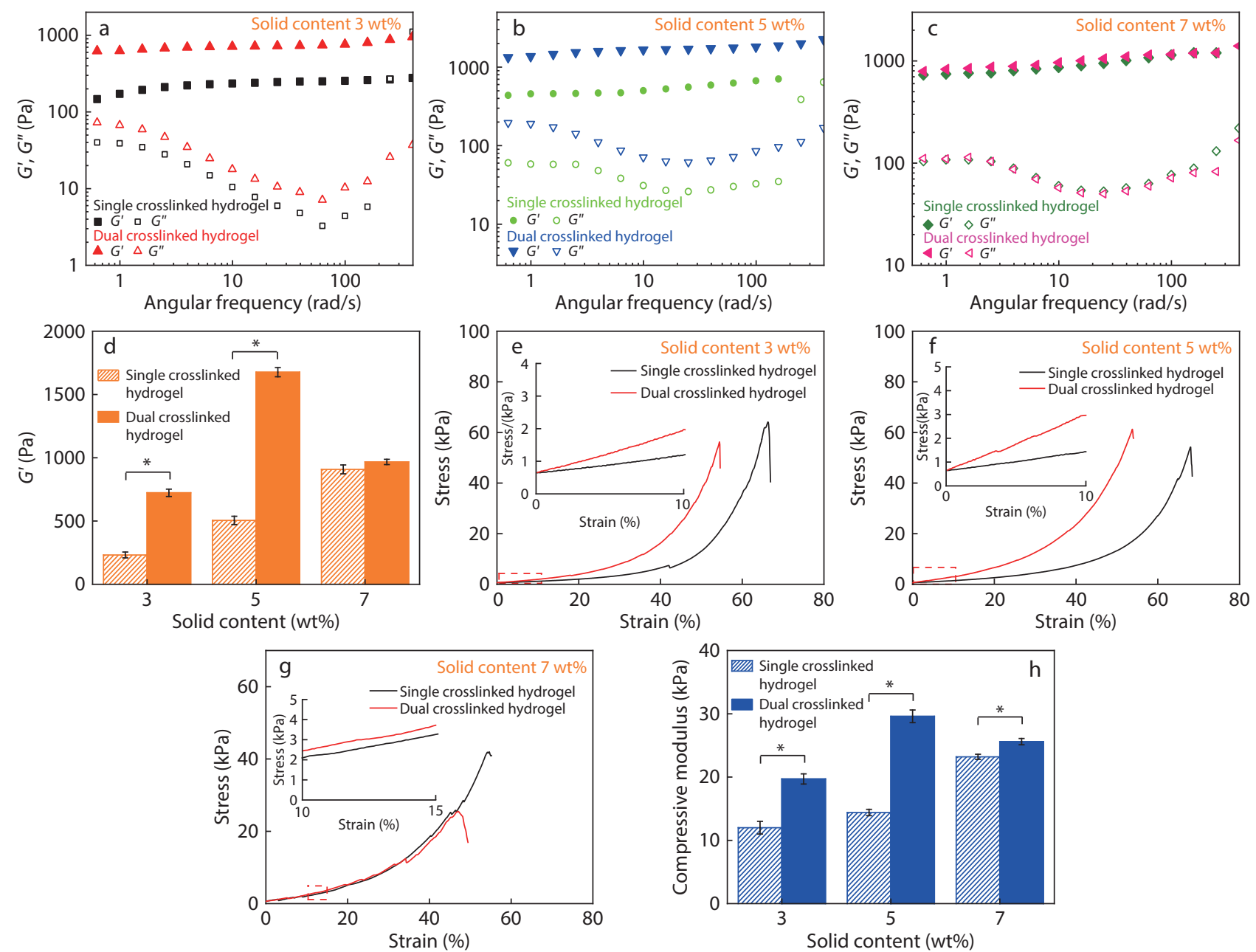

Fig. $3(\mathrm{a}-\mathrm{c})$ Frequency sweep of single and dual crosslinked hydrogels. (d) Storage moduli of single and dual crosslinked hydrogels at 10 rad/s. $(\mathrm{e}-\mathrm{g})$ Stress-strain curves of single and dual crosslinked hydrogels, and the inset is the partial enlarged image. (g) Compressive moduli of single and dual crosslinked hydrogels. $\left(n=3,{ }^{*} p<0.05\right)$. 
degradation behavior of $\mathrm{HA} / \gamma-\mathrm{PGA}$ hydrogel could also be further adjusted by UV light. Additionally, the single and dual crosslinked hydrogels with solid content of $7 \mathrm{wt} \%$ did not show significant difference in degradation time, which suggested that their crosslinking degree changed little before and after UV irradiation. The thermal stability of functionalized polymers as well as lyophilized $\mathrm{HA} / \mathrm{\gamma}$-PGA hydrogel was measured via TGA analysis. From the Fig. 2(h), the thermal decomposition temperatures of OM-HA, HM- $y-P G A$ and $\mathrm{HA} / \gamma^{-}$ PGA hydrogel were around 230,260 and $225^{\circ} \mathrm{C}$, respectively, which indicated that functionalized polymers and $\mathrm{HA} / \gamma-\mathrm{PGA}$ hydrogels had good thermostabilities to meet clinical requirements. Compared with the single crosslinked hydrogels, the dual crosslinked hydrogels showed a lower weight loss rate at $700{ }^{\circ} \mathrm{C}$, also indicating the formation of a more stable network structure. ${ }^{[39]}$

\section{Mechanical Properties of Hydrogels}

The mechanical properties of single and dual crosslinked hydrogels were examined by using rheological and compression tests. As shown in Figs. 3(a)-3(c), the $G^{\prime}$ values are slightly increased with the increase of angular frequency, and were larger than $G^{\prime \prime}$ by one order in magnitude, but the $G^{\prime}$ values were all lower than $2000 \mathrm{~Pa}$ (Fig. 3d), which indicated that both single and dual crosslinked HA/ $\gamma$-PGA hydrogels possessed a soft stable network. ${ }^{[40,41]}$ Compared with the $G^{\prime}$ of single crosslinked hydrogels, those of the dual crosslinked hydrogels exhibited a remarkable increase of about 3 times, indicating that the UV irradiation could further adjust the rheological properties of the single crosslinked hydrogels in a wide range. However, there was no significant difference in the values of $G$ ' between the single and dual crosslinked $\mathrm{HA} / \gamma$-PGA hydrogels with solid content of $7 w t \%$, which was consistent with the trends of swelling and degradation behaviors. This can be assumed that compared to the single crosslinked hydrogel with a low solid content, the high-concentration hydrogel has a higher crosslinking degree, and the polymer chains are difficult to stretch or move. When the high-concentration single crosslinked hydrogel is irradiated by UV light, the generated free radicals prefer to react with adjacent radicals and are difficult to react with the distant ones, so the crosslinking degree does not change much, thus showing a narrow range of property adjustment.

The anti-compression ability was evaluated by unconfined compression test. From the Figs. $3(\mathrm{e})-3(\mathrm{~g})$, the single crosslinked $\mathrm{HA} / \gamma_{-}-\mathrm{PGA}$ hydrogels with solid content of $3 \mathrm{wt} \%$ and $5 \mathrm{wt} \%$ could tolerate a stress of more than $50 \mathrm{kPa}$ and a strain
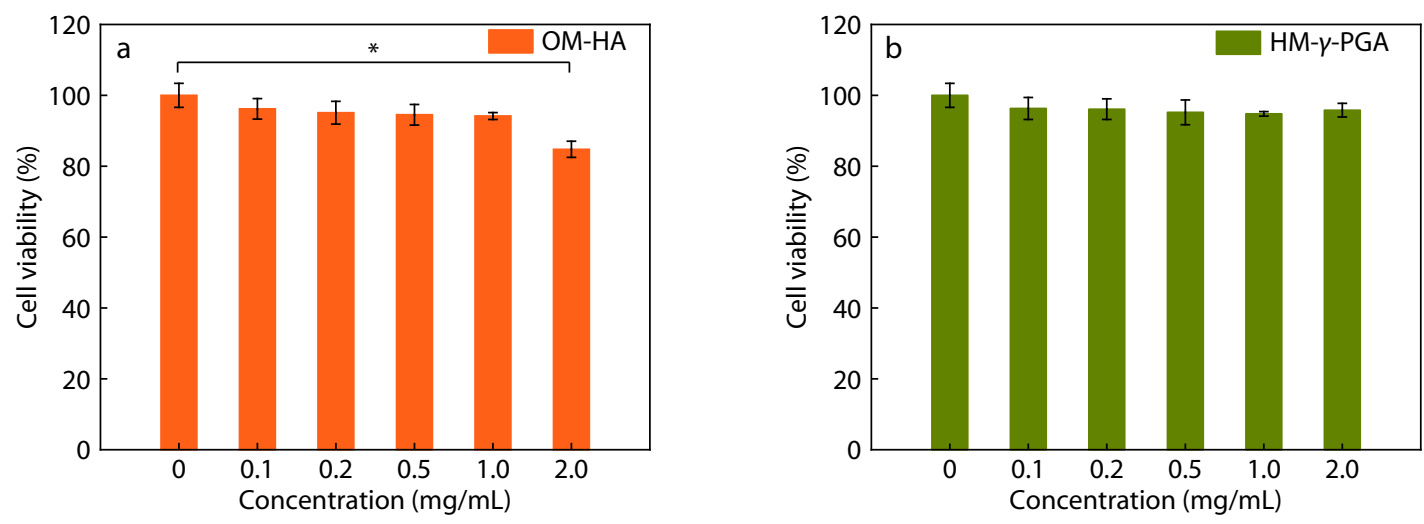

C

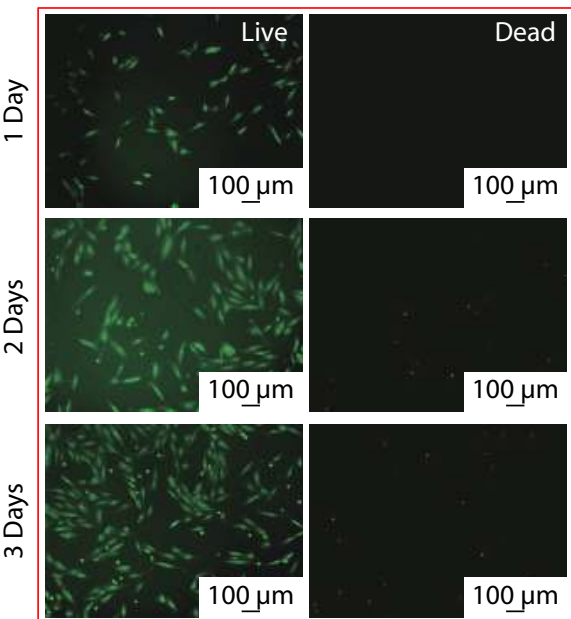

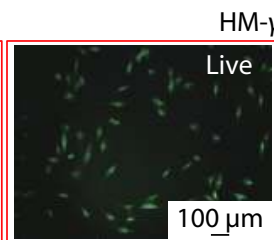
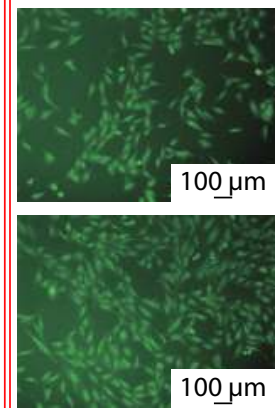
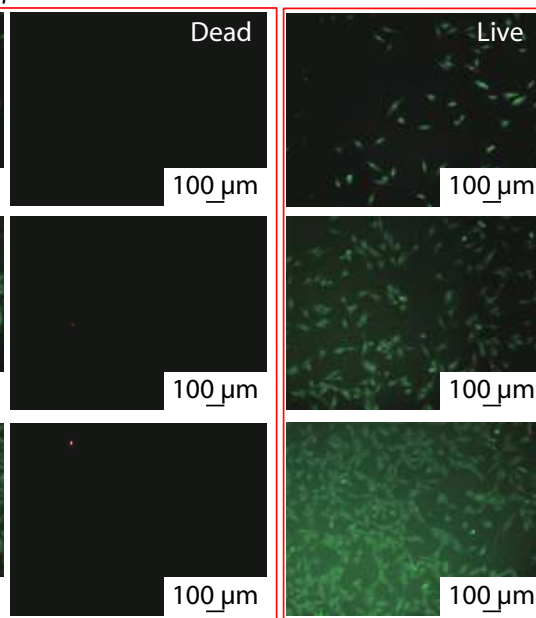

Control

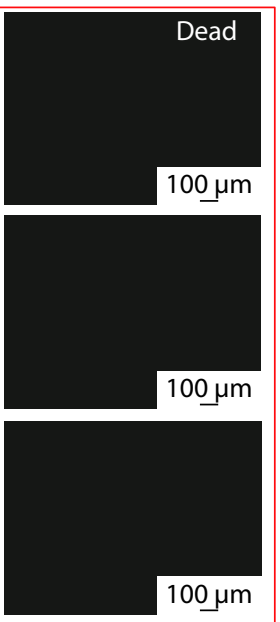

Fig. 4 Viability of NIH 3T3 cells cultured in different contents of (a) OM-HA solution and (b) HM- $\gamma$-PGA solution for $24 \mathrm{~h}$. (c) Fluorescence images of $\mathrm{NIH} 3 \mathrm{~T} 3$ cells cultured in functionalized polymer solutions $(2 \mathrm{mg} / \mathrm{mL})$. Cultures in medium without functionalized polymer were used as control. The living cells were green color and the dead cells were red color. $\left(n=3,{ }^{*} p<0.05\right)$. 
of around $70 \%$. Owing to the increase of crosslinking degree, the maximum fracture strains of high-concentration single crosslinked hydrogel and dual crosslinked hydrogels reduced, but could still reach about 50\%. As shown in Fig. 3(h), the dual crosslinked hydrogels showed a larger compressive modulus than that of the single crosslinked hydrogels. Especially, for the single crosslinked hydrogel with solid content of $5 \mathrm{wt} \%$, its compressive modulus increased from $14.4 \mathrm{kPa}$ to $29.6 \mathrm{kPa}$, which revealed that the UV irradiation could also further adjust the anti-compression ability of the single crosslinked hydrogels in a wide range. Additionally, the compressive moduli of single and dual crosslinked hydrogels were in kilopascal, which could meet the mechanical requirements of soft tissues. ${ }^{[22,43]}$ Therefore, the dynamic and static mechanical tests indicated that the developed $\mathrm{HA} / \mathrm{Y}$-PGA hydrogels had good potentials in soft tissue engineering.

\section{In Vitro Cytotoxicity and 3D Cell Culture}

The biocompatibility of precursor solutions is an important factor for the injectable biomaterials. Herein, the cytotoxicity of precursor solutions was examined by using MTT assay and live/dead analysis of NIH 3T3 cells. From the Figs. 4(a) and 4(b), the NIH 3 T3 cells cultured at various polymer concentrations exhibited a high cell viability; even at a high polymer concentration $(2.0 \mathrm{mg} / \mathrm{mL})$, the cell viability was still above $85 \%$. The fluorescence images corresponding to the live/dead analysis of NIH 3T3 cells cultured in functionalized polymer solutions were depicted in Fig. 4(c). The NIH 3T3 cells incubated with polymer solutions exhibited a spindle morphology similar to the control group, and with the increase of culture time, more green points were observed, which revealed the normal grow and proliferation of $\mathrm{NIH} 3 \mathrm{~T} 3$ cells in polymer solutions.

The 3D cell culture experiment was performed to explore the promising opportunities of $\mathrm{HA} / \gamma$-PGA hydrogels in tissue engineering as a cell scaffolding material. The 3D microscopic image of NIH 3T3 cells loaded in the dual crosslinked $\mathrm{HA} / \gamma^{-}$ PGA hydrogel is presented in Fig. 5. The hydrophilic nature and polyanionic structure of HA and $\gamma$-PGA enabled them as non-conducive materials towards cell adhesion, ${ }^{[44]}$ so the adhesive polypeptide RGD was introduced into the dual crosslinked $\mathrm{HA} / \gamma$-PGA hydrogels. The NIH $3 \mathrm{~T} 3$ cells loaded in the single crosslinked $\mathrm{HA} / \gamma$-PGA hydrogel was used as a control (Fig. S5 in ESI). After incubation for $48 \mathrm{~h}$, very few red fluorescent points could be found, which revealed the excellent cytocompatibility of $\mathrm{HA} / \gamma$-PGA hydrogels and tolerance of $\mathrm{NIH}$ 3T3 cells towards 3D encapsulation process of the dual
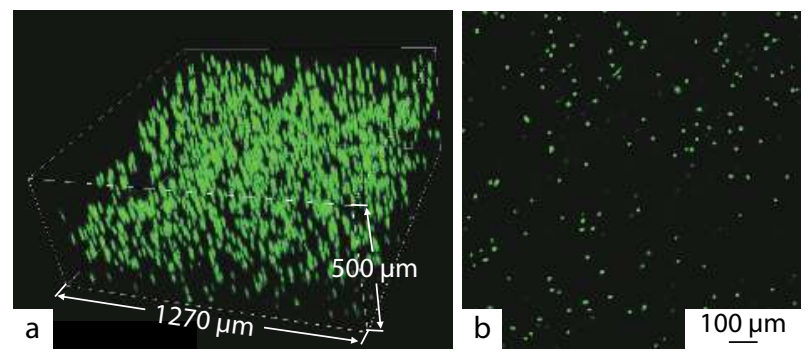

Fig. 5 (a) 3D confocal microscopy images and (b) their corresponding z-axis maximum projection images of NIH 3T3 cells in dual crosslinked hydrogel with solid content of $5 \mathrm{wt} \%$. The living cells were green color and the dead cells were red color. crosslinked $\mathrm{HA} / \mathrm{\gamma}$-PGA hydrogels. The above results confirmed the excellent cytocompatibility of OM-HA and $\mathrm{HM}-\gamma$ PGA solutions and the potential of $\mathrm{HA} / \gamma$-PGA hydrogels in tissue engineering.

\section{CONCLUSIONS}

In summary, we presented a dual crosslinking strategy for preparing injectable hydrogels with step-by-step tunable crosslinking degree by using Schiff base reaction and photopolymerization. The prepared $\mathrm{HA} / \mathrm{\gamma}-\mathrm{PGA}$ hydrogels had stepby-step tunable swelling, enzymatic degradation and mechanical properties, and compared with high-concentration $\mathrm{HA} / \gamma^{-}$ PGA hydrogels, the low-concentration $\mathrm{HA} / \gamma-\mathrm{PGA}$ hydrogels exhibited better adjustability. Moreover, the mechanical properties of $\mathrm{HA} / \gamma$-PGA hydrogels had a good match with soft tissue. In addition, the $\mathrm{HA} / \gamma-\mathrm{PGA}$ hydrogels fabricated from the biocompatible polymers (HA and $\gamma$-PGA) possessed excellent cytocompatibilities. Therefore, the developed HA/ $\gamma$-PGA hydrogels will be a promising candidate as injectable cell scaffold materials for soft tissue engineering.

\section{Electronic Supplementary Information}

Electronic supplementary information (ESI) is available free of charge in the online version of this article at http://dx.doi.org/10.1007/s10118-021-2558-3.

\section{ACKNOWLEDGMENTS}

This work was financially supported by the National Natural Science Foundation of China (No.31771049), Foundation of key R\&D Project of Jiangsu Province (No.BE2018731), Research Foundation of State Key Laboratory of Materials-Oriented Chemical Engineering (Nos.ZK201806 and No.KL18-06) and the Six Talent Peaks Project of Jiangsu Province (No.SWYY-046)

\section{REFERENCES}

1 Pertici, V.; Pin-Barre, C.; Rivera, C.; Pellegrino, C.; Laurin, J.; Gigmes, D.; Trimaille, T. Degradable and injectable hydrogel for drug delivery in soft tissues. Biomacromolecules 2019, 20, 149-163.

2 Qu, J.; Zhao, X.; Liang, Y.; Xu, Y.; Ma, P. X.; Guo, B. Degradable conductive injectable hydrogels as novel antibacterial, antioxidant wound dressings for wound healing. Chem. Eng. J. 2019, 362, 548-560.

3 Jung, Y. S.; Park, W.; Park, H.; Lee, D. K.; Na, K. Thermo-sensitive injectable hydrogel based on the physical mixing of hyaluronic acid and Pluronic F-127 for sustained NSAID delivery. Carbohydr. Polym. 2017, 156, 403-408.

4 Yan, S.; Wang, T.; Feng, L.; Zhu, J.; Zhang, K.; Chen, X.; Cui, L.; Yin, J. Injectable in situ self-cross-linking hydrogels based on poly(Lglutamic acid) and alginate for cartilage tissue engineering. Biomacromolecules 2014, 15, 4495-4508.

5 Fu, S.; Dong, H.; Deng, X.; Zhuo, R.; Zhong, Z. Injectable hyaluronic acid/poly(ethylene glycol) hydrogels crosslinked via strain-promoted azide-alkyne cycloaddition click reaction. Carbohydr. Polym. 2017, 169, 332-340. 
6 Yan, S.; Wang, T.; Li, X.; Jian, Y.; Zhang, K.; Li, G.; Yin, J. Fabrication of injectable hydrogels based on poly(L-glutamic acid) and chitosan. RSC Adv. 2017, 7, 17005-17019.

7 Carvalho, I. C.; Mansur, H. S. Engineered 3D-scaffolds of photocrosslinked chitosan-gelatin hydrogel hybrids for chronic wound dressings and regeneration. Mater. Sci. Eng. C-Mater. Biol. Appl. 2017, 78, 690-705.

8 Kang, W.; Bi, B.; Zhuo, R.; Jiang, X. Photocrosslinked methacrylated carboxymethyl chitin hydrogels with tunable degradation and mechanical behavior. Carbohydr. Polym. 2017, $160,18-25$.

9 Bian, L.; Hou, C.; Tous, E.; Rai, R.; Mauck, R. L.; Burdick, J. A. The influence of hyaluronic acid hydrogel crosslinking density and macromolecular diffusivity on human MSC chondrogenesis and hypertrophy. Biomaterials 2013, 34, 413-421.

10 Oommen, O. P.; Wang, S.; Kisiel, M.; Sloff, M.; Hilborn, J.; Varghese, O. P. Smart design of stable extracellular matrix mimetic hydrogel: synthesis, characterization, and in vitro and in vivo evaluation for tissue engineering. Adv. Funct. Mater. 2013, 23, 1273-1280.

11 Wei, Z.; Yang, J. H.; Liu, Z. Q.; Xu, F.; Zhou, J. X.; Zrínyi, M.; Osada, Y.; Chen, Y. M. Novel biocompatible polysaccharide-based selfhealing hydrogel. Adv. Funct. Mater. 2015, 25, 1352-1359.

12 Pandit, A. H.; Mazumdar, N.; Ahmad, S. Periodate oxidized hyaluronic acid-based hydrogel scaffolds for tissue engineering applications. Int. J. Biol. Macromol. 2019, 137, 853-869.

$13 \mathrm{Ma}, \mathrm{X}_{\text {; }} \mathrm{Xu}$, T.; Chen, W.; Qin, H.; Chi, B.; Ye, Z. Injectable hydrogels based on the hyaluronic acid and poly $(\gamma$-glutamic acid) for controlled protein delivery. Carbohydr. Polym. 2018, 179, 100-109.

14 Zou, Y. J.; He, S. S.; Du, J. Z. ع-Poly(L-lysine)-based hydrogels with fast-acting and prolonged antibacterial activities. Chinese $J$. Polym. Sci. 2018, 36, 1239-1250.

15 Duan, J. J.; Zhang, L. N. Robust and smart hydrogels based on natural polymers. Chinese J. Polym. Sci. 2017, 35, 1165-1180.

16 Shi, L.; Zhao, Y.; Xie, Q.; Fan, C.; Hilborn, J.; Dai, J.; Ossipov, D. A. Moldable hyaluronan hydrogel enabled by dynamic metalbisphosphonate coordination chemistry for wound healing. Adv. Healthc. Mater. 2017, 1700973.

17 Bian, S.; He, M.; Sui, J.; Cai, H.; Sun, Y.; Liang, J.; Fan, Y.; Zhang, X. The self-crosslinking smart hyaluronic acid hydrogels as injectable three-dimensional scaffolds for cells culture. Colloid Surf. B-Biointerfaces 2016, 140, 392-402.

18 Mohandas, A.; Anisha, B. S.; Chennazhi, K. P.; Jayakumar, R. Chitosan-hyaluronic acid/VEGF loaded fibrin nanoparticles composite sponges for enhancing angiogenesis in wounds. Colloid Surf. B-Biointerfaces 2015, 127, 105-113.

19 Hwang, H. D.; Cho, H. J.; Balakrishnan, P.; Chung, C. W.; Yoon, I. S.; Oh, Y. K.; Byun, Y.; Kim, D. D. Cross-linked hyaluronic acid-based flexible cell delivery system: application for chondrogenic differentiation. Colloid Surf. B-Biointerfaces 2012, 91, 106-113.

20 Collins, M. N.; Birkinshaw, C. Hyaluronic acid based scaffolds for tissue engineering-a review. Carbohydr. Polym. 2013, 92, 1262-1279.

21 Pescosolido, L.; Schuurman, W.; Malda, J.; Matricardi, P.; Alhaique, F.; Coviello, T.; van, Weeren P. R.; Dhert, W. J.; Hennink, W. E.; Vermonden, T. Hyaluronic acid and dextran-based semi-IPN hydrogels as biomaterials for bioprinting. Biomacromolecules 2011, 12, 1831-1838.

22 Murphy, S. V.; Skardal, A.; Atala, A. Evaluation of hydrogels for bio-printing applications. J. Biomed. Mater. Res. Part A 2013, 101, 272-284.

23 Gentilini, C.; Dong, Y.; May, J. R.; Goldoni, S.; Clarke, D. E.; Lee, B. H.; Pashuck, E. T.; Stevens, M. M. Functionalized poly( $\gamma$-glutamic acid) fibrous scaffolds for tissue engineering. Adv. Healthc. Mater. 2012, 1, 308-315.

24 Zhang, D.; Feng, X.; Zhou, Z.; Zhang, Y.; Xu, H. Economical production of poly $(\gamma$-glutamic acid) using untreated cane molasses and monosodium glutamate waste liquor by Bacillus subtilis NX-2. Bioresour. Technol. 2012, 114, 583-588.

25 Clarke, D. E.; Pashuck, E. T.; Bertazzo, S.; Weaver, J. V. M.; Stevens, M. M. Self-healing, self-assembled $\beta$-sheet peptide-poly $(\gamma-$ glutamic acid) hybrid hydrogels. J. Am. Chem. Soc. 2017, 139, 7250-7255.

26 Xu, T.; Yang, R.; Ma, X.; Chen, W.; Liu, S.; Liu, X.; Cai, X.; Xu, H.; Chi, B. Bionic poly( $\gamma$-glutamic acid) electrospun fibrous scaffolds for preventing hypertrophic scars. Adv. Healthc. Mater. 2019, 1900123.

27 Yang, R.; Wang, X.; Liu, S.; Zhang, W.; Wang, P.; Liu, X.; Ren, Y.; Tan, $\mathrm{X}_{\text {; }} \mathrm{Chi}$, B. Bioinspired poly( $\gamma$-glutamic acid) hydrogels for enhanced chondrogenesis of bone marrow-derived mesenchymal stem cells. Int. J. Biol. Macromol. 2020, 142, 332-344

28 Zhuang, H.; Hong, Y.; Gao, J.; Chen, S.; Ma, Y.; Wang, S. A poly( $\gamma-$ glutamic acid)-based hydrogel loaded with superoxide dismutase for wound healing. J. Appl. Polym. Sci. 2015, 42033.

29 Ito, T.; Yeo, Y.; Highley, C. B.; Bellas, E.; Benitez, C. A.; Kohane, D. S. The prevention of peritoneal adhesions by in situ cross-linking hydrogels of hyaluronic acid and cellulose derivatives. Biomaterials 2007, 28, 975-983.

30 Huh, H. W.; Zhao, L.; Kim, S. Y. Biomineralized biomimetic organic/inorganic hybrid hydrogels based on hyaluronic acid and poloxamer. Carbohydr. Polym. 2015, 126, 130-140.

31 Li, L.; Wang, N.; Jin, X.; Deng, R.; Nie, S.; Sun, L.; Wu, Q.; Wei, Y.; Gong, C. Biodegradable and injectable in situ cross-linking chitosan-hyaluronic acid based hydrogels for postoperative adhesion prevention. Biomaterials 2014, 35, 3903-3917.

32 Li, N. N.; Fu, C. P.; Zhang, L. M. Using casein and oxidized hyaluronic acid to form biocompatible composite hydrogels for controlled drug release. Mater. Sci. Eng. C-Mater. Biol. Appl. 2014, 36, 287-293.

33 Dooling, L. J.; Buck, M. E.; Zhang, W. B.; Tirrell, D. A. Programming molecular association and viscoelastic behavior in protein networks. Adv. Mater. 2016, 28, 4651-4657.

34 Tomer, R.; Dimitrijevic, D.; Florence., A. T. Electrically controlled release of macromolecules from cross-linked hyaluronic acid hydrogels. J. Control. Release 1995, 33, 405-413.

35 Kunioka, M.; Choi, H. J. Hydrolytic degradation and mechanical properties of hydrogels prepared from microbial poly(amino acid)s. Polym. Degrad. Stabil. 1998, 59, 33-31.

36 Jeon, O.; Song, S. J.; Lee, K. J.; Park, M. H.; Lee, S. H.; Hahn, S. K.; Kim, S.; Kim, B. S. Mechanical properties and degradation behaviors of hyaluronic acid hydrogels cross-linked at various cross-linking densities. Carbohydr. Polym. 2007, 70, 251-257. 
37 Jiao, Y.; Liu, Z.; Zhou, C. Fabrication and characterization of PLLAchitosan hybrid scaffolds with improved cell compatibility. J. Biomed. Mater. Res. Part A 2007, 80, 820-825.

38 Yuan, L.; Wu, Y.; Gu, Q. S.; El-Hamshary, H.; El-Newehy, M.; Mo, X. Injectable photo crosslinked enhanced double-network hydrogels from modified sodium alginate and gelatin. Int. J. Biol. Macromol. 2017, 96, 569-577.

39 Zhao, Y.; Ma, L.; Zeng, R.; Tu, M.; Zhao, J. Preparation, characterization and protein sorption of photo-crosslinked cell membrane-mimicking chitosan-based hydrogels. Carbohydr. Polym. 2016, 151, 237-244.

40 Martínez-Ruvalcaba, A.; Chornet, E.; Rodrigue, D. Viscoelastic properties of dispersed chitosan/xanthan hydrogels. Carbohydr.
Polym. 2007, 67, 586-595.

41 Bai, H.; Sheng, K.; Zhang, P.; Li, C.; Shi, G. Graphene oxide/conducting polymer composite hydrogels. J. Mater. Chem. 2011, 21, 18653-18658.

42 Engler, A. J.; Sen, S.; Sweeney, H. L.; Discher, D. E. Matrix elasticity directs stem cell lineage specification. Cell 2006, 126, 677-689.

43 Bootsma, K.; Fitzgerald, M. M.; Free, B.; Dimbath, E.; Conjerti, J.; Reese, G.; Konkolewicz, D.; Berberich, J. A.; Sparks, J. L. 3D printing of an interpenetrating network hydrogel material with tunable viscoelastic properties. J. Mech. Behav. Biomed. Mater. 2017, 70, 84-94.

44 Rose, S. F.; Lewis, A. L.; Hanlon, G. W.; Lloyd, A. W. Biological responses to cationically charged phosphorylcholine-based materials in vitro. Biomaterials 2004, 25, 5125-5135. 